\title{
A Generalization of Hadwiger's Transversal Theorem to Intersecting Sets
}

\author{
Rephael Wenger \\ McGill University, 805 Sherbrooke Street West, Montreal, Quebec H3A 2K6, Canada
}

\begin{abstract}
Given an ordered family of compact convex sets in the plane, if every three sets can be intersected by some directed line "consistent" with the ordering, then there exists a common transversal of the family. This generalizes Hadwiger's Transversal Theorem to families of compact convex sets which are not necessarily pairwise disjoint. If every six sets can be intersected by some directed line "consistent" with the ordering, then there exists a common transversal which is "consistent" with the ordering. If the family is pairwise disjoint and every four sets can be intersected by some directed line "consistent" with the ordering, then there exists a common transversal which is "consistent" with the ordering.
\end{abstract}

\section{Introduction}

Let $\boldsymbol{A}$ be a family of compact convex sets in the plane. A common transversal for $A$ is a line which intersects every one of the elements of $A$. Given some ordering of the elements of $A$ a directed line $l$ is consistent with that ordering if, for every pair of disjoint sets $a$ and $b$ intersected by $l, a$ precedes $b$ in the ordering if and only if $l$ intersects $a$ before $b$. A common transversal is consistent with some ordering if one of the two directed lines corresponding to the common transversal is consistent with that ordering.

Hadwiger proved that given an ordered family of pairwise disjoint compact convex sets in the plane, if every three sets can be intersected by some directed line consistent with the ordering then there exists a common transversal of the family [3], [4]. We generalize this result by dropping the condition that the sets must be pairwise disjoint. In so doing we offer a new proof of Hadwiger's Transversal Theorem.

Hadwiger's Transversal Theorem says nothing about the order in which the common transversal will intersect the family. We give necessary and sufficient conditions for the existence of a common transversal with a specific ordering. 


\section{Generalizations of Hadwiger's Transversal Theorem}

If a directed line intersects a pair of compact convex sets, then we say that the direction of the line is a stabbing direction for the pair. A stabbing direction induces an ordering on a pair of disjoint compact convex sets, namely the order in which any directed line with the given direction stabs the pair.

Lemma 1. There exists a common transversal for a family of compact convex sets in the plane if and only if there exists some direction which is a stabbing direction for every two sets. There exists a common transversal for a family of compact convex sets in the plane which is consistent with a given ordering if and only if there exists some direction which is a stabbing direction for every two sets and the induced ordering on disjoint sets is consistent with the given ordering.

Proof. Assume that there exists some direction which is a stabbing direction for every two sets. Project the sets onto a line which is orthogonal to the stabbing direction. The projection of each set is a closed line segment and every pair of these line segments intersect. By Helly's Theorem there exists some point in the intersection of all the line segments [1]. The line through this point with the given stabbing direction is a common transversal for the entire family.

Now assume that there exists some direction which is a stabbing direction for every two sets and the induced ordering on disjoint sets is consistent with the given ordering. By the argument above, there exists a directed line with the given stabbing direction which stabs the family. Every two disjoint sets are stabbed by some translate of this directed line in the given order so the directed line must stab every two disjoint sets consistent with the given ordering.

A line separates two convex sets if each convex set lies in a different closed half-plane defined by the line. A line strictly separates two convex sets if it separates the two sets and does not intersect either of the sets. If a directed line $l$ strictly separates two convex sets, $a, b$, then we say that the direction of the line is a separation direction for the pair. We say that $a$ is to the left of $l$ if $a$ appears to the left when facing in the direction $l$. Each separation direction can be associated with an ordering of $a b, a$ to the left of $b$, or $b a, b$ to the left of $a$.

Lemma 2. Any direction is either a stabbing direction or a separation direction for a pair of compact convex sets but not both.

We leave the proof to the reader. Both Lemmas 1 and 2 generalize easily to conditions for the existence of hyperplane stabbers in higher dimensions. In fact, Lemma 1 can be generalized to a condition for $k$-flat stabbing in $R^{d}$.

Directions in the plane can be mapped to points on a circle. The stabbing directions of two intersecting compact convex sets map to the entire circle. The stabbing directions of two disjoint compact convex sets, $a, b$, map to two disjoint arcs on the circle. Each of these arcs can be associated with a different ordering of the sets, either $a b$ or $b a$. The separation directions also map to two disjoint 
arcs on the circle. Each of these arcs can be associated with a different ordering of the sets, either $a b$ or $b a$, representing $a$ to the left of $b$ or $b$ to the left of $a$. Lemma 2 states that the circle is covered by the four arcs representing stabbing and separation directions and the intersection of any two of these arcs is disjoint. By combining Lemmas 1 and 2 we get the following corollary.

Corollary 1. Given a family of compact convex sets in the plane, if there is some direction which is not a separation direction for any pair of sets, then there exists a common transversal for the family.

Finally, we have our generalization of Hadwiger's Transversal Theorem.

Theorem 1. Given an ordered family of compact convex sets in the plane, if every three sets can be intersected by some directed line consistent with the ordering, then there exists a common transversal of the family.

Proof. Let $A$ be the family of compact convex sets. Let $S$ be the set of all arcs corresponding to separation directions for every pair of disjoint sets in $A$. Corollary 1 is equivalent to saying if the arcs in $S$ do not cover the circle, then there exists a common transversal of $A$.

We can associate with each arc in $S$ a unique label $a b$ or $b a$ depending on whether $a$ or $b$ is to the left of separating lines with the given direction. Let $S_{1}$ be the set of all arcs labeled $a b$ where $a$ precedes $b$ in the given ordering and let $S_{2}$ be the set of all other arcs.

We claim that the intersection of any two arcs in $S_{1}$ and $S_{2}$ is empty. Let $a b$ be some arc in $S_{1}$ and let $y x$ be an arc in $S_{2}$. Let $l$ be a line separating $a$ from $b$ and let $l^{\prime}$ be a parallel line separating $y$ from $x$. Assume $l$ lies to the left of $l^{\prime}$. $a$ lies to the left of $l$ and $b$ and $x$ to the right. $x$ lies to the right of $l^{\prime}$ and $a$ and $y$ to the left. Convex sets $a$ and $x$ are distinct, since $x$ and $a$ are separated by a line. $x$ cannot come before $a$ in the ordering because a line which intersected $x$, $a, b$ in that order would cross $l$ twice. $a$ cannot come before $x$ in the ordering because a line which intersected $y, x, a$ in that order would cross $l^{\prime}$ twice. By a similar argument, $l$ cannot lie to the right of $l^{\prime}$ or coincide with $l^{\prime}$. It follows that the intersection of $a b$ and $y x$ is empty.

Assume all the arcs in $S$ cover the circle. $S$ must not be empty, so $S_{1}$ and $S_{2}$ must not be empty. Let $P_{i}$ be the union of all the arcs in $S_{i}, i=1,2$. $P_{1}$ and $P_{2}$ are open nonempty sets which cover the circle. Since the circle is connected, $P_{1}$ intersects $P_{2}$. This contradicts the previous argument that no arc in $S_{1}$ intersects an arc in $S_{2}$. We conclude that $S$ does not cover the circle. Thus there exists some direction such that every pair of sets in $A$ are stabbed in that direction. By Lemma 1 , there exists a common transversal for $A$.

Hadwiger et al. used a similar argument to prove Proposition 27 in [4].

To prove Theorem 2 we use the following well-known theorem. If every three arcs in a family of circular arcs have a point in common and every arc is smaller than a semicircle, then all the arcs of the family have a common point. For a proof, see Proposition 17 in [4]. 
Theorem 2. Given an ordered family of at least six compact convex sets in the plane, if every six sets can be intersected by some directed line consistent with the ordering, then there exists a common transversal consistent with the ordering.

Proof. Let $S$ be the set of all arcs corresponding to stabbing directions for pairs of disjoint sets which are consistent with the given ordering. Each arc has measure less than $180^{\circ}$ and every three arcs intersect, so the arcs have a common point. Lemma 1 gives a common transversal consistent with the given ordering.

Theorem 3. Given an ordered family of at least four pairwise disjoint compact convex sets in the plane, if every four sets can be intersected by some directed line consistent with the ordering, then there exists a common transversal consistent with the ordering.

Proof. We first show that Theorem 3 is true for a family of five sets. Let $\{a, b, c, d, e\}$ be a family of pairwise disjoint compact convex sets in the plane with the alphabetic ordering. For every four sets there is a directed line stabber consistent with the alphabetic order. Let $p_{a b c d}$ be the point on the circle corresponding to the stabber of $a b c d$. In the same manner define points $p_{a b c e}, p_{a b d e}$, $p_{a c d e}$, and $p_{b c d e}$. Any three of these points are covered by some arc corresponding to a stabbing direction for a pair of ordered sets. For instance $p_{a b c d}, p_{a b c e}$, and $p_{a b d e}$ are covered by the arc corresponding to the stabbing directions for $a b$. Thus every three of these points must lie within some half-circle. If the center of the circle lies within the convex hull of these five points, then it lies within the convex hull of three of the points by Caratheodory's theorem [1], and these three points would not lie in a half-circle. It follows that all the points must lie in some half-circle $\theta$.

Let $S$ be the set of all arcs corresponding to stabbing directions for pairs of disjoint sets which are consistent with the alphabetic ordering. Intersect each of the arcs in $S$ with the half-circle $\theta$ to form the set $S^{\prime}$. The intersection of each pair of arcs in $S^{\prime}$ contains one of the five points. By Helly's theorem and Lemma 1 , the intersection of all the arcs is nonempty and there exists a stabber consistent with the alphabetic ordering.

We now show that Theorem 3 is true for a family of six sets, $\{a, b, c, d, e, f\}$ where every four sets have a stabber consistent with the alphabetic ordering. By the argument above, every five of the sets have a stabber consistent with the alphabetic ordering. Choose the points $p_{a b c d e}, p_{a b c d f}, p_{a b c e f}, p_{a b d e f}, p_{a c d e f}$, and $p_{b c d e f}$ corresponding to the six stabbers. By the same argument as before, all these points lie in some half-circle $\theta$, the pairs of arcs corresponding to stabbing directions intersect in $\theta$ and there is a stabber consistent with the alphabetic ordering.

Given any ordered family of pairwise disjoint compact convex sets in the plane we just showed that if every four sets can be intersected by some directed line consistent with the given ordering, then every six sets can be so intersected. By Theorem 2 , there exists a common transversal for the entire family consistent with the given ordering. 


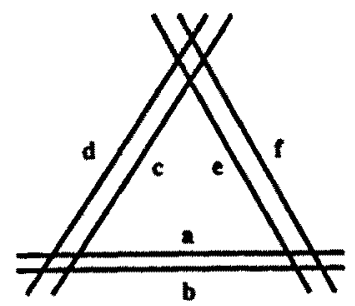

Fig. 1. Six line segments with no common transversal in order abcdef.

The numbers four and six in Theorems 2 and 3 are tight. Figure 1 contains an example of a family of line segments where every five can be intersected consistent with the alphabetic ordering but no six can. Figure 2 contains an example of a family of pairwise disjoint line segments where every three can be intersected by a line consistent with the given ordering while no four can.

\section{Generalizing the Generalization}

The restriction in Hadwiger's Transversal Theorem to families of convex sets can be changed to a restriction to families of connected sets. Given a family of compact connected sets in the plane and some ordering of the sets, if every three sets can be intersected by a directed line consistent with the ordering then there exists a common transversal of the family. In this case, a directed line $l$ is consistent with an ordering if, for every pair of sets $a$ and $b$ intersected by $l$ whose convex hulls are disjoint, $a$ prcedes $b$ in the ordering if and only if $l$ intersects $a$ before $b$. The proofs remain exactly the same.

Katchalski generalized Hadwiger's Transversal Theorem to the following:

Given an ordered family of pairwise disjoint compact convex sets in $R^{d}$, if every three sets can be intersected by a line consistent with the ordering then there exists a hyperplane which intersects each member of the family [5].

Applying the same exact arguments for generalizing Hadwiger's Transversal Theorem, we can eliminate the pairwise disjointness condition in Katchalski's

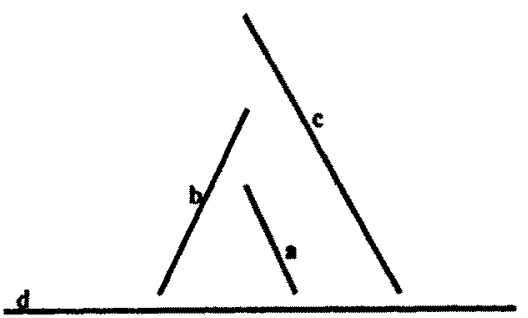

Fig. 2. Four line segments with no common transversal in order abcd. 
theorem. This generalization was found with the help of Richard Pollack. Jacob E. Goodman and Richard Pollack observed that the arguments would allow an even more general statement of Katchalski's theorem, namely:

Given an ordered family of compact convex sets in $R^{d}$ and a connected, centrally symmetric region $\Theta$ on the hypersphere in $R^{d}$, if every three sets can be intersected by a line consistent with the ordering then there exists a hyperplane which intersects each member of the family with a normal in $\Theta$.

Pollack and Goodman generalized Hadwiger's Transversal Theorem to provide necessary and sufficient conditions for the existence of a hyperplane transversal in $d$-space [2]. Their generalization included the condition that no $d$ members of the family were intersected by a $d-2$-fiat. It is an open question whether this condition can be removed as it was for Hadwiger's Transversal Theorem and Katchalski's theorem.

\section{Acknowledgments}

I would like to thank David Avis and Richard Pollack for their advice and help in writing this paper. I also thank the referees for eliminating some special case analysis from Theorem 1.

\section{References}

1. Danzer, L., Grünbaum, B., and Klee, V., Helly's theorem and its relatives, in Convexity, 100-181, Proceedings of Symposia in Pure Mathematics, Vol. 7, American Mathematical Society, Providence, RI, 1963.

2. Goodman, H. E. and Pollack, R., Hadwiger's transversal theorem in higher dimensions, J. Amer. Math. Soc. 1 (2) (1988).

3. Hadwiger, H., Über Eibereiche mit gemeinsamer Treffgeraden, Portugal Math. 16 (1957), 23-29.

4. Hadwiger, H., Debrunner, H., and Klee, V., Combinatorial Geometry in the Plane, Holt, Rinehart, and Winston, New York, 1964.

5. Katchalski, M., Thin sets and common transversals, J. Geom. 14 (1980), 103-107.

Received October 26, 1987, and in revised form March 30, 1988.

Note added in proof. Richard Pollack and Rephael Wenger recently generalized Hadwiger's Transversal Theorem to necessary and sufficient conditions for the existence of a hyperplane transversal in $d$-space. Their result is valid for all families of compact convex sets, solving the open problem mentioned above. 